\title{
Erratum to: Connectedness properties of the space of complete nonnegatively curved planes
}

\author{
Igor Belegradek ${ }^{1} \cdot \operatorname{Jing} \mathrm{Hu}^{1}$
}

Published online: 4 January 2016

(C) Springer-Verlag Berlin Heidelberg 2016

\section{Erratum to: Math. Ann. (2015) 362:1273-1286 DOI 10.1007/s00208-014-1159-7}

In Theorem 4.1 of the original article we claimed that a certain parametrization of the space of complete nonnegatively curved metrics on $\mathbb{R}^{2}$ was a homeomorphism, but the spaces were topologized incorrectly. The correct statement of Theorem 4.1 can be obtained by fixing any number $\alpha$ with $0<\alpha<1$ and replacing every entry of $k$ with $k+\alpha$, i.e., the bijection (1.1) of the original article given by $(\phi, u) \rightarrow \phi^{*} e^{-2 u} g_{0}$ is a homeomorphism if $\phi$ varies in the $C^{k+1+\alpha}$ topology while $u$ and $\phi^{*} e^{-2 u} g_{0}$ vary in the $C^{k+\alpha}$ topology. The discussion of Theorem 4.1 in the introduction to the original article must be modified accordingly.

The proof of Theorem 4.1 works as originally written once every entry of $k$ is replaced by $k+\alpha$. Instead of basic facts about $C^{k}$ maps one uses standard properties of Hölder spaces, see [1, Section 4.1] and [2, Section 2.2]. The mistake was due to the incorrect application of the Continuity Theorem proved in [3] for the $C^{k+\alpha}$ topology rather than the $C^{k}$ topology. We suspect that the statement of Theorem 4.1 in the original article is false but have no counterexamples.

This change affects no other results in the original article because they only use continuity of the bijection (1.1) when $\phi$ and $u$ vary in the $C^{\infty}$ topology and $\phi^{*} e^{-2 u} g_{0}$ varies in the $C^{k}$ topology.

The online version of the original article can be found under doi:10.1007/s00208-014-1159-7.

Igor Belegradek

ib@math.gatech.edu

Jing $\mathrm{Hu}$

jhu61@math.gatech.edu

1 School of Mathematics, Georgia Institute of Technology, Atlanta, GA 30332-0160, USA 


\section{References}

1. Gilbarg, D., Trudinger, N.S.: Elliptic partial differential equations of second order. In: Classics in Mathematics. Springer, Berlin (2001). (Reprint of the 1998 edition)

2. Bojarski, B., Hajlasz, P., Strzelecki, P.: Sard's theorem for mappings in Hölder and Sobolev spaces. Manuscripta Math. 118(3), 383-397 (2005)

3. Earle, C.J., Schatz, A.: Teichmüller theory for surfaces with boundary. J. Differ. Geom. 4, 169-185 (1970) 